\title{
Should we be worried about the NHS selling patient data?
}

The original final sentence in this Feature (BMJ 2020;368:m113, doi:10.1136/bmj.m113) was ambiguous and has been removed. 